\title{
ARTICLE OPEN \\ Selection rules for Cooper pairing in two-dimensional interfaces and sheets
}

\author{
Mathias S. Scheurer ${ }^{1}$, Daniel F. Agterberg ${ }^{2}$ and Jörg Schmalian ${ }^{1,3}$
}

Thin sheets deposited on a substrate and interfaces of correlated materials offer a plethora of routes towards the realization of exotic phases of matter. In these systems, inversion symmetry is broken which strongly affects the properties of possible instabilities -in particular in the superconducting channel. By combining symmetry and energetic arguments, we derive general and experimentally accessible selection rules for Cooper instabilities in noncentrosymmetric systems, which yield necessary and sufficient conditions for spontaneous time-reversal-symmetry breaking at the superconducting transition and constrain the orientation of the triplet vector. We discuss in detail the implications for various different materials. For instance, we conclude that the pairing state in thin layers of $\mathrm{Sr}_{2} \mathrm{RuO}_{4}$ must, as opposed to its bulk superconducting state, preserve time-reversal symmetry with its triplet vector being parallel to the plane of the system. All triplet states of this system allowed by the selection rules are predicted to display topological Majorana modes at dislocations or at the edge of the system. Applying our results to the $\mathrm{LaAlO}_{3} / \mathrm{SrTiO}_{3}$ heterostructures, we find that while the condensates of the (001) and (110) oriented interfaces must be time-reversal symmetric, spontaneous time-reversal-symmetry breaking can only occur for the less studied (111) interface. We also discuss the consequences for thin layers of $\mathrm{URu}_{2} \mathrm{Si}_{2}$ and $\mathrm{UPt}_{3}$ as well as for single-layer FeSe. On a more general level, our considerations might serve as a design principle in the search for time-reversal-symmetry-breaking superconductivity in the absence of external magnetic fields.

npj Quantum Materials (2017)2:9; doi:10.1038/s41535-016-0008-1

\section{INTRODUCTION}

The realization and characterization of two-dimensional (2D) superconducting phases in various different systems constitutes a topic of great current interest. ${ }^{1-5}$ This is motivated by the promising role played by $2 \mathrm{D}$ superconductors in the search for topological Majorana modes and related applications, ${ }^{6}$ by the tunability of the electronic properties, ${ }^{2,4,7-9}$ and by the fundamental interest in superconducting transitions in reduced dimensions. Particularly interesting examples are given by $\mathrm{LaAlO}_{3} / \mathrm{SrTiO}_{3}$ heterostructures, that show very rich electronic behavior, ${ }^{10}$ and single-layer FeSe on [001] $\mathrm{SrTiO}_{3}$ with significantly enhanced transition temperatures compared to its bulk value. ${ }^{3}$ This also motivates closer inspection of superconducting thin films of other correlated materials such as $\mathrm{Sr}_{2} \mathrm{RuO}_{4}{ }^{11}$ and $\mathrm{UPt}_{3}{ }^{12}$ which, in addition, promises to offer insights into the electronic properties of the bulk material.

Noncentrosymmetric 2D superconductors form a particularly important class since inversion symmetry is naturally broken in the practical realization of 2D systems: As shown in Fig. 1 a, both for an interface as well as for a thin layer on a substrate ( $B$ is vacuum) or in an asymmetric environment ( $B$ not vacuum, but $A \neq B$ ), the system is not invariant under the transformation $\boldsymbol{r} \rightarrow-\boldsymbol{r}$ of the three-dimensional (3D) spatial coordinates; inversion symmetry can only be restored in the case of a thin layer in a symmetric environment as shown in Fig. 1b.

A pivotal property of superconducting states is their behavior under the inversion of the time direction. Not only does it determine the topological classification ${ }^{13}$ but also essentially influences the electromagnetic and thermal responses of these systems. $^{14}$

In this work, constraints on possible pairing states of noncentrosymmetric systems are derived that follow from the combination of symmetry and energetic arguments. These "symergetic" selection rules state that

(I) if a superconductor has an order parameter that transforms according to a complex or multi-dimensional irreducible representation (IR) of the point group $\mathcal{G}$ of the normal state, it must break time-reversal symmetry (TRS). Note that this is not generally true in systems with inversion symmetry (see, e.g., refs 15 and 16 ).

(II) In 2D, TRS can only be broken in systems with threefold rotation symmetry.

(III) In the presence of a twofold rotation symmetry $C_{2}^{\perp}$ perpendicular to the plane of a 2D system, the component of the triplet vector along the axis of $C_{2}^{\perp}$ must vanish.

These results hold under the assumption that (i) the energetic splitting $E_{\text {so }}$ of the Fermi surfaces is larger than the superconducting order parameter and (ii) that the superconducting phase does not break translation invariance. As we discuss in detail below, statement (II) follows from the fact that the superconducting pairing potential $\tilde{\Delta}_{\mathbf{k}}=\left\langle\phi_{\mathbf{k a}}\left|\Delta_{\mathbf{k}} T^{\dagger}\right| \phi_{\mathbf{k a}}\right\rangle$ for singly degenerate Fermi surface $a$ (with associated Bloch wavefunctions $\phi_{\mathbf{k} a}$, microscopic superconducting order parameter $\Delta_{\boldsymbol{k}}$, see Eq. (2), and the unitary part $T$ of the antiunitary time-reversal operator $\Theta$ ) is even under $\boldsymbol{k} \rightarrow-\boldsymbol{k}$ with $2 \mathrm{D}$ momentum $\boldsymbol{k}$-a result that was

\footnotetext{
${ }^{1}$ Institute for Theory of Condensed Matter Physics, Karlsruhe Institute of Technology (KIT), Karlsruhe 76131, Germany; ${ }^{2}$ Department of Physics, University of Wisconsin-Milwaukee, Milwaukee, WI 53211, USA and ${ }^{3}$ Institute for Solid State Physics, Karlsruhe Institute of Technology (KIT), Karlsruhe 76131, Germany

Correspondence: Mathias S. Scheurer (mscheurer@tkm.uni-karlsruhe.de)
}

Received: 12 July 2016 Revised: 22 November 2016 Accepted: 6 December 2016

Published online: 16 February 2017 
(a)

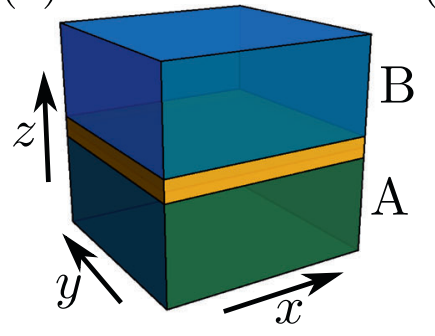

(b)

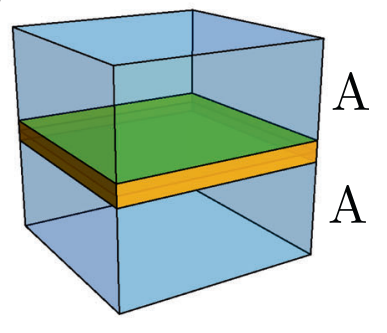

(c)

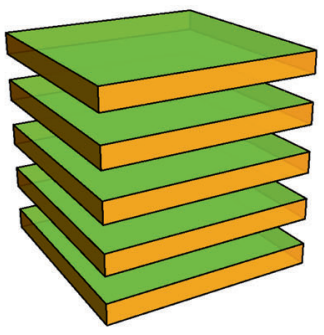

Fig. 1 From a symmetry point of view, 2D systems (yellow) can be grouped into those realized in an asymmetric (a) or symmetric (b) environment. Layered materials consisting of weakly coupled sheets as shown in (c) are not included in our definition of 2D systems

(a)

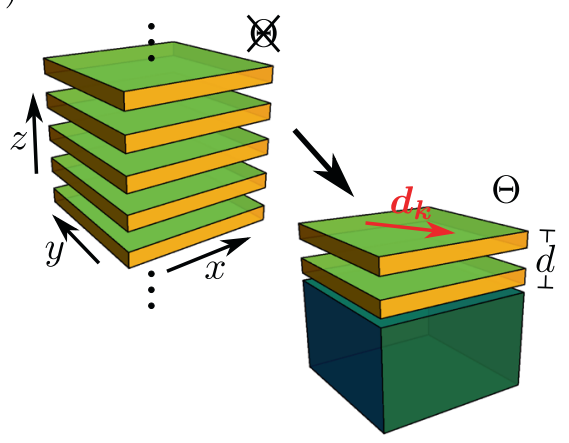

(c)

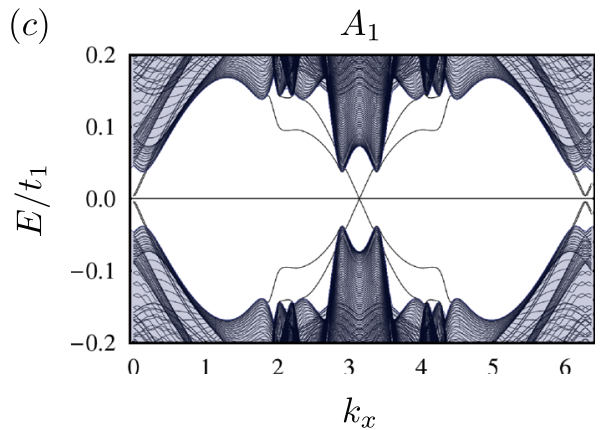

(b)

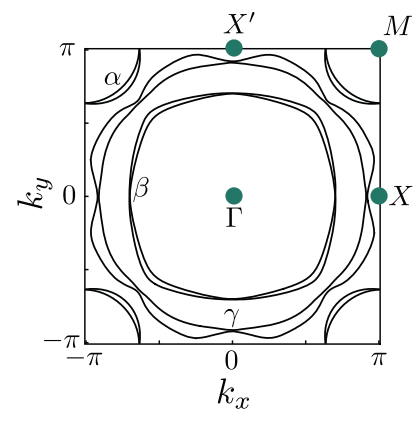

(d)

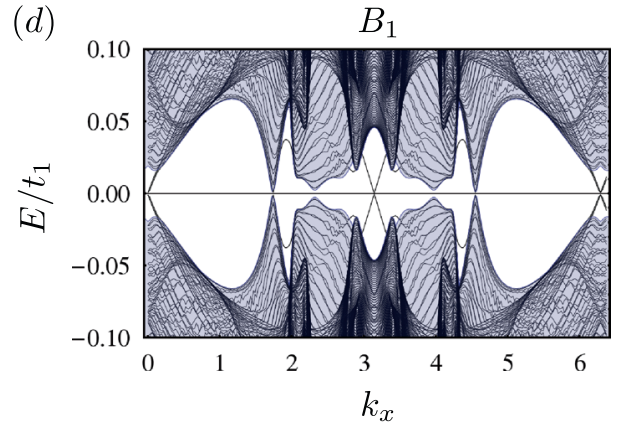

Fig. 2 a Illustration of the gedankenexperiment explained in the main text with superconductor and substrate shown in yellow and green, respectively. b Fermi surfaces of thin layers of $\mathrm{Sr}_{2} \mathrm{RuO}_{4}$ following from the model defined in the "Methods" section. c and d show the lowenergy part of the spectrum of the superconducting states transforming under $A_{1}$ and $B_{1}$ for open (periodic) boundary conditions along the $y$ $(x)$ direction. For concreteness, we have taken pure triplet pairing with $\mathbf{d} \propto 0.2 t_{1}\left(\sin k_{y},-\sin k_{x}, 0\right)^{T}$ and $\mathbf{d} \propto 0.08 t_{1}\left(\sin k_{y}, \sin k_{x}, 0\right)^{T}$, respectively (cf. Table 1). As argued in the main text, the spectra of the $B_{2}$ and $A_{2}$ pairing states look qualitatively similar to (c) and (d)

obtained in ref. 17 We emphasize that our notion of 2D does not include strongly anisotropic 3D systems illustrated in Fig. 1c.

In the remainder of the paper, we will first discuss the consequences for the time-reversal and topological properties of possible pairing states in several different $2 \mathrm{D}$ materials and then present a proof of the selection rules (I), (II), and (III).

\section{RESULTS}

The selection rules stated above naturally lead to the gedankenexperiment illustrated in Fig. 2a: imagine putting a 3D bulk superconductor with a TRS-breaking order parameter on a substrate and gradually reducing its thickness $d$. If the resulting, necessarily noncentrosymmetric, point group $\mathcal{G}$ of the thin layer system does not contain a threefold rotation symmetry, either superconductivity disappears or TRS must be restored below a critical value of $d$. Furthermore, if $C_{2}^{\perp} \in \mathcal{G}$, the triplet vector must be aligned parallel to the plane of the system.
Consequences for $\mathrm{Sr}_{2} \mathrm{RuO}_{4}$

A natural candidate material for this gedankenexperiment is provided by $\mathrm{Sr}_{2} \mathrm{RuO}_{4}$ hosting a superconducting phase ${ }^{18}$ which is widely believed to be a TRS-breaking chiral $p$-wave state with triplet vector $\boldsymbol{d}_{\boldsymbol{k}} \propto\left(k_{x}+i k_{y}\right) \boldsymbol{e}_{z} \cdot{ }^{19-21}$ Due to its small superconducting transition temperature and the near degeneracy ${ }^{22}$ of the chiral $p$-wave order parameter with the triplet states transforming under the 1D IRs of its bulk point group $D_{4 h r}{ }^{23}$ along with the strong spin-orbit coupling of Ru (following from the large atomic number $Z=44$ ), we expect the selection rules to apply. Furthermore, thin layers of this material have been fabricated and shown to be superconducting. ${ }^{11}$ As the point group is reduced to $C_{4 v}$ by the presence of the substrate, we conclude that both TRS must be restored and the triplet vector must rotate to be aligned in the plane of the system upon reducing the thickness $d$. We note that these general predictions are confirmed by the explicit singleband-model calculations in refs 24,25 .

Since the superconducting condensate belongs to symmetry class DIII, ${ }^{13}$ it is characterized by a $Z_{2}$ topological invariant $v$ with 
Table 1. Possible pairing states for a system with $C_{4 v}$ point group such as thin layers of $\mathrm{Sr}_{2} \mathrm{RuO}_{4}$

\begin{tabular}{lllll}
\hline Gr. th. & Pairing & Symmetry & TRS & $d k \cdot \sigma$ \\
\hline$A_{1}$ & s-wave & $1, X^{2}+Y^{2}$ & $y$ & $Y \sigma_{x}-X \sigma_{y}$ \\
$A_{2}$ & $g$-wave & $X Y\left(X^{2}-Y^{2}\right)$ & $y$ & $X \sigma_{x}+Y \sigma_{y}$ \\
$B_{1}$ & $d_{x^{2}-y^{2}}$ & $X^{2}-Y^{2}$ & $y$ & $Y \sigma_{x}+X \sigma_{y}$ \\
$B_{2}$ & $d_{x y}$ & $X Y$ & $y$ & $X \sigma_{x}-Y \sigma_{y}$ \\
$E(1,0)$ & $e_{(1,0)}$ & $X$ & $y$ & $\sigma_{z} Y$ \\
$E(1,1)$ & $e_{(1,1)}$ & $X+Y$ & $y$ & $\sigma_{z}(Y-X)$ \\
$E(1, i)$ & $e_{(1, i)}$ & $X+i Y$ & $n$ & $\sigma_{z}(X+i Y)$ \\
\hline
\end{tabular}

It is indicated whether the phase preserves $(y)$ or breaks $(n)$ TRS. Here $X$ and $Y$ are continuous functions on the whole Brillouin zone transforming as the in-plane momenta $k_{x}$ and $k_{y}$. To define the triplet component of the order parameter, we use $\sigma_{j}$ to denote Pauli matrices in spin space. As shown in the main text, the state transforming under $E$, with triplet vector along $z$, is energetically disfavored if (i) holds. Although our analysis is more general, we here focus, for simplicity, on pairing states that transform trivially in orbital/subband space.

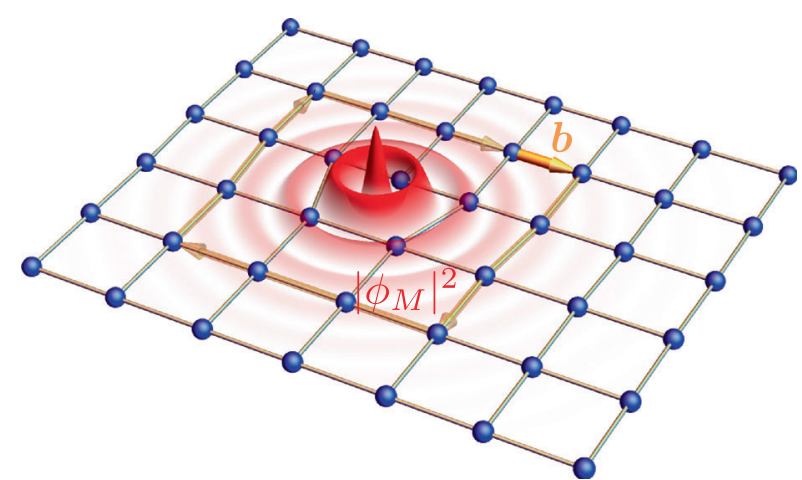

Fig. 3 If the superconducting state in thin layers of $\mathrm{Sr}_{2} \mathrm{RuO}_{4}$ is fully gapped, a Kramers pair of Majorana modes (red) will be localized at dislocations charactered by a Burgers vector $\boldsymbol{b}$ (orange) with $b_{1}+b_{2}$ odd

$v=-1 \quad(v=+1)$ defining the topological (trivial) phase. ${ }^{6}$ The invariant is given by ${ }^{26}$

$\nu=\prod_{a}\left(\operatorname{sign}\left(\tilde{\Delta}_{\boldsymbol{k}_{a} a}\right)\right)^{m_{a}}$,

where the product involves all Fermi surfaces, $\boldsymbol{k}_{\mathrm{a}}$ is an arbitrary momentum on $a$ and $m_{a}$ the number of time-reversal invariant momenta (green dots in Fig. 2b) enclosed by Fermi surface $a$. In order to calculate the topological properties of the pairing states that are not excluded by our selection rules, i.e., those transforming under the four 1D IRs of $C_{4 v}$ (see Table 1), we take the tight-binding model for the $\mathrm{Ru} t_{2 g}$ states with atomic spinorbit coupling that is commonly used ${ }^{27}$ for bulk $\mathrm{Sr}_{2} \mathrm{RuO}_{4}$ (see "Methods" section) adding the inversion asymmetric hopping term $\delta\left(L_{x} \sin \left(k_{y}\right)-L_{y} \sin \left(k_{x}\right)\right)$ allowed by the residual $C_{4 v}$ symmetry. Here $L_{j}$ denote angular momentum operators projected onto the $t_{2 g}$ manifold. The prefactor $\delta$ is nonuniversal and unknown, however, the following discussion is independent of its value as long as all $m_{a}$ are the same as in the limit $\delta=0$ which holds as long as $\delta<0.47 t_{1}$ with $t_{1}$ denoting the largest centrosymmetric hopping parameter. The resulting Fermi surfaces for the rather large value $\delta=0.45 t_{1}$ are shown in Fig. 2b.

In addition, we assume that the triplet component is larger than the admixed singlet component since triplet is dominant in bulk $\mathrm{Sr}_{2} \mathrm{RuO}_{4}$. Consequently, the sign of $\tilde{\Delta}_{\boldsymbol{k}_{a} a}$ is opposite on Fermi surfaces split by the inversion asymmetric hopping $\delta \neq 0$. From
Eq. (1) and Fig. 2b it then directly follows that the (gapless) state transforming under $A_{1}$ is topological. This is confirmed by the spectrum shown in Fig. 2c characterized by a gapless Kramers pair of Majorana modes crossing the bulk gap in the vicinity of $k_{x}=\pi$. The subgap states around $k_{x}=0$ result from the $\beta$ and $\gamma$ bands being topological separately. The band mixing of the superconducting pairing, however, introduces a mass to the associated edge modes. Since Eq. (1) also holds for the 1D DIII $Z_{2}$ invariant (with $\left.m_{\mathrm{a}}=1\right){ }^{26}$ we directly find the nontrivial invariant $v_{\mathrm{x}}=-1\left(v_{\mathrm{y}}\right.$ $=-1)$ for the fictitious 1D system at $k_{\mathrm{x}}=\pi\left(k_{\mathrm{y}}=\pi\right)$. Therefore, the system is characterized by the weak indices $(1,1)$ such that a Kramers doublet of isolated Majorana modes emerges at any dislocation with Burgers vector $\boldsymbol{b}=\left(b_{1}, b_{2}\right)$ satisfying $b_{1}+b_{2}$ odd $^{28,29}$ as illustrated in Fig. 3.

The orbital polarization and symmetry restrictions render the Fermi-surface splitting very small along the high-symmetry lines $k_{\mathrm{x}}=0, \pi$ and $k_{\mathrm{y}}=0, \pi$ as can be seen in Fig. $2 \mathrm{~b}$. Although being nodal in the limit $\Delta \ll E_{\mathrm{so}}$, the $B_{2}$ pairing state is thus fully gapped for values of the order parameter that are larger than the small splitting at these high-symmetry lines but much smaller than the average value of $E_{\text {so }}$ such that the symergetic selection rules still apply. For this reason, the $B_{2}$ state has the same topological signatures as the $A_{1}$ order parameter.

However, the $B_{1}$ and $A_{2}$ states have nodes along the $\Gamma-\mathrm{M}$ direction and, hence, the topological invariant $v$ of the 2D system is ill-defined. Nonetheless, the nontrivial fictitious 1D invariant $v_{x}$ implies the presence of Majorana modes around $k_{x}=\pi$ at an interface parallel to the $x$ axis which is confirmed by Fig. 2d. This only holds as long as translation symmetry is preserved along $x$ which is not a very constraining assumption as very clean samples are already required to stabilize the superconducting state itself.

Taken together, this discussion implies that thin films of $\mathrm{Sr}_{2} \mathrm{RuO}_{4}$ represent a promising setup for the realization of Majorana modes and that the latter might be helpful in determining the pairing state of the 2D system which could provide insights for the bulk material as well.

\section{Other TRS-breaking condensates}

Let us discuss two further materials, $\mathrm{URu}_{2} \mathrm{Si}_{2}$ and $\mathrm{UPt}_{3}$, which are believed to host a TRS-breaking superconducting bulk state. ${ }^{30,31}$

To begin with $\mathrm{URu}_{2} \mathrm{Si}_{2}$, we first note that it is from a symergetic point of view very similar to $\mathrm{Sr}_{2} \mathrm{RuO}_{4}$ : The point group $D_{4 h}$ of its bulk is reduced to $C_{4 v}$ in a thin film of (001) orientation and the combination of the strong spin-orbit coupling of $U(Z=92)$ and the small transition temperature ${ }^{32}$ makes assumption (i) plausible. It follows that, if the thin film still displays superconductivity, TRS must be restored in the condensate and the triplet component of the order parameter must be aligned parallel to the plane of the system. More specifically, from a pure symmetry point of view, the most natural ${ }^{33}$ candidate pairing state is $e_{(1, i)}$ transforming under the IR $E$ subduced from $E_{g}$ of $D_{4 h}$, which is the IR of the bulk pairing state $\Delta_{\boldsymbol{k}}=i \sigma_{y}\left(k_{x}+i k_{y}\right) k_{z} \cdot{ }^{30,34}$ Since this state is suppressed if assumption (i) holds, superconductivity is likely to disappear in the thin-layer system, which, in addition, implies that inversionsymmetry breaking impurities are expected to be strongly pair breaking.

As for $\mathrm{UPt}_{3}$, the selection rules are expected to be applicable for the same reason as in the case of $\mathrm{URu}_{2} \mathrm{Si}_{2}$ and $\mathrm{Sr}_{2} \mathrm{RuO}_{4}$, but the point symmetries are different: The bulk point group $D_{6 h}$ is reduced to $C_{6 v}$ in a (001) film and, hence, contains a threefold rotation symmetry such that TRS-breaking cannot be excluded. If superconductivity does not disappear, $C_{2}^{\perp} \in C_{6 v}$ implies that the triplet vector, which is largely aligned along the $z$ axis in the bulk condensate, ${ }^{35}$ must rotate to be parallel to the $x y$ plane. We note in this context that superconducting (001)-oriented films of $\mathrm{UPt}_{3}$ have been reported in ref. 12 From a symmetry point of view, there are ten possible superconducting phases-four associated 
with the four 1D IRs of $C_{6 v}$ and three with each of the two 2D IRs $E_{1}$ and $E_{2}$. Since they are odd under $\boldsymbol{k} \rightarrow-\boldsymbol{k}$, we exclude $E_{1}$ and two of the 1D IRs. Furthermore, the two time-reversal symmetric order parameter configurations transforming under $E_{2}$ can be discarded [see (I)] and we are left with only three candidate pairing states for the thin layer system: The time-reversal symmetric $s$-wave and $i$ wave states transforming under $A_{1}$ and $A_{2}$, respectively, as well as the TRS-breaking state transforming as $(X+i Y)^{2}$.

\section{Further examples}

Finally, let us discuss three additional 2D superconducting systems without TRS-breaking 3D analog. We begin with the $\mathrm{LaAlO}_{3} / \mathrm{SrTiO}_{3}$ heterostructures that show interface conductivity ${ }^{36-38}$ for the three different orientations (001), (110), and (111) of the interface with respective point groups $C_{4 v}, C_{2 v}$, and $C_{3 v}$ while superconductivity has so far only been reported for the former two orientations. ${ }^{1,5}$ Even though the proton number of $\mathrm{Ti}$ is smaller than that of the atoms relevant to the previously discussed materials, the experimentally reported strong spin-orbit splitting of the Fermi surfaces ${ }^{39,40}$ shows that assumption (i) is clearly appropriate. From result (II), it follows that the condensates of the (001) and (110) interfaces must be necessarily time-reversal symmetric whereas the (111) heterostructure allows for exotic TRS-breaking superconductivity. Due to the absence of a $C_{2}^{\perp}$ symmetry it is also the first system we have discussed so far that makes an out-of-plane triplet vector possible [see (III)]. Taken together this motivates a closer experimental inspection of the low-temperature properties of the (111) interface.

Another 2D system with threefold symmetry, that is a candidate for TRS breaking, is $\mathrm{MoS}_{2}$ that was shown to be superconducting via ion gating. 4,8

In order to calculate the topological invariant $v$ in Eq. (1) of the (001) and (110) oxide interfaces, a microscopic calculation has to be performed since there is no 3D analog to compare with and the symmetry properties alone do not determine $v$. The analysis of refs 41,42 shows that the topological properties are directly related to the origin (electron-phonon/purely electronic) of the interaction driving the superconducting instability.

Our final example is single-layer $\mathrm{FeSe}$ on $\mathrm{SrTiO}_{3}$. If assumption (i) is also valid for this system, the symergetic restrictions apply and the superconductor cannot transform under the $2 \mathrm{D}$ IR of $C_{4 v}$, thus, preserving TRS. In combination with experiment indicating the absence of nodes, ${ }^{43}$ there are only three possible pairing states: The pairing field can have the same sign on all four (spin-split) electron pockets around the $M$ point $\left(s^{++++}\right)$, the signs can be pairwise identical $\left(s^{++-}\right)$or only differ on one Fermi surface $\left(s^{+++-}\right)$. Only the latter pairing state has a nontrivial DIII invariant $v$ as readily follows from Eq. (1). It has recently been shown ${ }^{42}$ under very general assumptions that an $\mathrm{s}^{+++-}$state is not possible irrespective of whether superconductivity arises from the coupling to collective particle-hole modes or from phonons. Therefore, FeSe is most likely a topologically trivial, TRS-preserving superconductor.

\section{Spontaneous symmetry breaking}

After having illustrated the consequences for several different materials, we will next proof the selection rules (I), (II), and (III). In order to decide which superconducting states are possible, we consider a system with pairing Hamiltonian

$H_{\mathrm{MF}}=\sum_{\boldsymbol{k}}\left[\psi_{\boldsymbol{k}}^{\dagger} h_{\boldsymbol{k}} \psi_{\boldsymbol{k}}+\frac{1}{2}\left(\psi_{\boldsymbol{k}}^{\dagger} \Delta_{\boldsymbol{k}}\left(\psi_{-\boldsymbol{k}}^{\dagger}\right)^{T}+\right.\right.$ H.c. $\left.)\right]$,

already taking into account assumption (ii). The fermionic creation and annihilation operators $\psi_{\boldsymbol{k}}{ }^{\dagger}$ and $\psi_{\boldsymbol{k}}$ are $N$-component spinors that describe the spin and orbital degrees of freedom as well as potentially relevant subbands that result from the confinement along the direction perpendicular to the plane of the system. Correspondingly, the normal state Hamiltonian $h_{\boldsymbol{k}}$ and the pairing function $\Delta_{\boldsymbol{k}}$ in Eq. (2) are $N \times N$ matrices. Note that this approach and the following analysis go beyond the pseudospin description that is commonly used ${ }^{17,44,45}$ for studying pairing in systems with spin-orbit coupling.

To begin with the constraints resulting from symmetries, let us investigate the transformation properties of $\Delta_{\boldsymbol{k}}$ under timereversal and the elements $g$ of the point group $\mathcal{G}$ of the normal state. Time-reversal acts on the pairing field according to

$\Delta_{\boldsymbol{k}} \stackrel{\ominus}{\rightarrow} T \Delta_{-\boldsymbol{k}}^{*} T^{T}$.

Under a point group operation $g \in \mathcal{G}$ holds $\Delta_{\boldsymbol{k}} \stackrel{g}{\rightarrow} R_{\psi}(g) \Delta_{R_{v}^{-1}(g) \mathbf{k}} R_{\psi}^{T}(g)$, where $R_{v}(g)$ and $R_{\psi}(g)$ transform vectors and spinors, respectively.

To identify the order parameter, we expand the pairing field

$\Delta_{\mathbf{k}}=\sum_{n} \sum_{\mu=1}^{d_{n}} \eta_{\mu}^{n} X_{\boldsymbol{k} \mu}^{n}$

with respect to the basis of $N \times N$ matrix fields $X_{\boldsymbol{k} \mu}^{n}$ transforming under the IR $n$ of $\mathcal{G}$. Here $d_{n}$ is the dimensionality of the IR $n$ and $\eta_{\mu}^{n}$ are complex-valued coefficients. Note that, before analyzing fluctuations, we first have to determine the form and, in particular, the symmetry properties of possible order parameters which is the central theme of the present work. Including fluctuations will modify the behavior of physical quantities in the vicinity of the phase transition. As will be seen below, the superconducting transitions we investigate are always second order on the meanfield level and, hence, a Ginzburg-Landau (GL) expansion can be used to determine the candidate pairing states. Taking into account the usual orthogonality relations of $\mathrm{IRs}^{23}$ the free energy $F$ assumes the form

$F\left[\eta_{\mu}^{n}\right]=F[0]+\sum_{n} \sum_{\mu=1}^{d_{n}} a_{n}(T)\left|\eta_{\mu}^{n}\right|^{2}+F_{\geq 4}\left[\eta_{\mu}^{n}\right]$

with higher order terms $F_{\geq 4} \in \mathcal{O}\left(|\eta|^{4}\right)$. The coefficient $a_{n_{0}}(T)$ that first changes sign determines the IR $n=n_{0}$ of the order parameter. If the representation $n_{0}$ is real, the TRS of the normal state implies that the matrix fields $X_{\boldsymbol{k} \mu}^{n_{0}} T^{\dagger}$ can be chosen to be Hermitian (see Supplementary Information S1) and from Eq. (3) follows $\eta_{\mu}^{n_{0} \stackrel{\ominus}{\rightarrow}} \pm\left(\eta_{\mu}^{n_{0}}\right)^{*}$ for $\Theta^{2}=\mp 1$. As the global phase of the order parameter can always be absorbed by a $U(1)$ transformation of the fields, we need at least a $2 \mathrm{D}\left(d_{n_{0}}>1\right)$ order parameter vector with a nontrivial relative phase to break TRS. For instance, in the case of $C_{4 v}{ }^{23}$ summarized in Table 1, only the pairing state $e_{(1, i)}$ transforming under the 2D IR $E$ breaks TRS. Note that this is different in the case of complex representations, where time-reversed partners transform according to different IRs. Consequently, we have to identify pairing states either in complex or in multi-dimensional IRs to obtain a TRS-breaking superconductor.

Weak-pairing limit

To deduce the consequences resulting from the energetic assumption (i), it is convenient to diagonalize the free Hamiltonian $h_{\boldsymbol{k}}$ by the unitary transformation $\psi_{\boldsymbol{k} i}=\Sigma_{a}\left(\phi_{\mathbf{k} a}\right)_{i} f_{\mathbf{k a}}$ that is made of its eigenfunctions $\phi_{\mathbf{k} a}$ satisfying $h_{\boldsymbol{k}} \phi_{\mathbf{k} a}=\varepsilon_{\mathbf{k} a} \phi_{\mathbf{k} a}$. Since $h_{\boldsymbol{k}}$ is timereversal symmetric, i.e. $\Theta h_{\boldsymbol{k}} \Theta^{-1}=h_{-\boldsymbol{k}}$, we know that $\Theta \phi_{\mathbf{k} a}$ is an eigenstate of $h_{-\boldsymbol{k}}$ with the same energy. The broken inversion symmetry at the interface along with spin-orbit coupling further imply that the Fermi surfaces are non-degenerate in the generic case. This implies for the wave functions that ${ }^{46}$

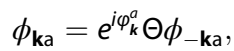

where the phase factors must satisfy the condition $e^{i \varphi_{k}^{a}}=\mp e^{i \varphi_{-k}^{a}}$ as a consequence of $\Theta^{2}=\mp 1$. The Hamiltonian can now be cast in 


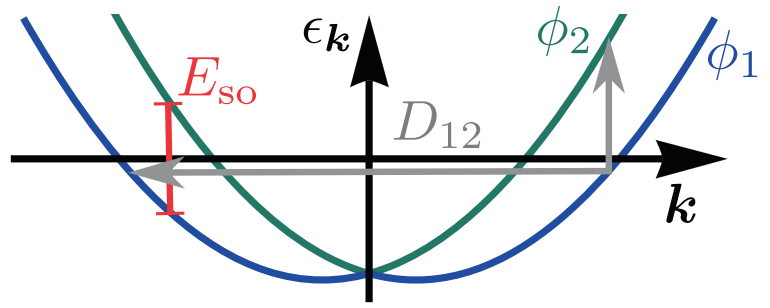

Fig. 4 Due to assumption (i), the inter-Fermi surface matrix elements (gray arrow) can be neglected as they couple states separated by energies of order $E_{\mathrm{so}}$

the quadratic form $H=1 / 2 \sum_{k} \Psi_{k}^{\dagger} h_{k}^{\text {BdG }} \Psi_{\mathbf{k}}$ using the Nambu spinor $\Psi_{\mathbf{k a}}=\left(f_{\mathbf{k a}}, f_{-\mathbf{k a}}^{\dagger} e^{-i \varphi_{k}^{a}}\right)^{T}$. The off-diagonal elements of the associated Bogoliubov-de Gennes (BdG) Hamiltonian, characterizing the superconducting state, are given by $D_{\mathbf{k a b}}=\left\langle\phi_{\mathbf{k a}}\left|\Delta_{\boldsymbol{k}} T^{\dagger}\right| \phi_{\mathbf{k b}}\right\rangle$.

We now consider the weak-pairing limit (see Fig. 4) that implies that partners of a Cooper pair always originate within a given Fermi sheet and not between states of different sheets. If this is the case, it holds

$D_{\boldsymbol{k a b}}=\tilde{\Delta}_{\mathbf{k a}} \delta_{\mathrm{ab}}$.

Note, this assumption does not exclude frequently discussed pairing states that are due to interband interactions. It merely requires that anomalous averages are made of the same quantum numbers as the normal state. In this weak-pairing limit, we immediately obtain the eigenvalues of the BdG Hamiltonian $h_{k}$ BdG as $E_{\mathbf{k a}}= \pm\left(\varepsilon_{\mathbf{k a}}^{2}+\left|\tilde{\Delta}_{\mathbf{k a}}\right|^{2}\right)^{1 / 2}$, i.e. $\left|\tilde{\Delta}_{\mathbf{k} a}\right|$ is the superconducting gap on the Fermi surface.

The behavior of $\tilde{\Delta}_{\mathbf{k} a}$ under point group operations follows from inserting Eq. (4) and using that the wave functions of non-degenerate Fermi surfaces must transform as $\phi_{\mathbf{k a}}=$

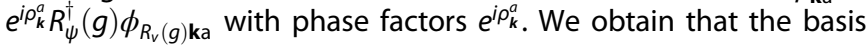
functions $\varphi_{\mathbf{k a}}^{\mu n}:=\left\langle\phi_{\mathbf{k a}}\left|X_{\mathbf{k} \mu}^{n} T^{\dagger}\right| \phi_{\mathbf{k a}}\right\rangle$ transform under the same, $a$-independent, IRs as the matrix fields $X_{\boldsymbol{k} \mu}^{n}$. Thus, once we have found the IR $n_{0}$ under which the pairing field $\Delta_{\boldsymbol{k}}$ transforms, along with the associated order parameter vector $\boldsymbol{\eta}^{n_{0}}=\left(\boldsymbol{\eta}_{1}^{n_{0}}, \cdots, \boldsymbol{\eta}_{d_{n_{0}}}^{n_{0}}\right)$, we also know the symmetry properties of the gap function

$\tilde{\Delta}_{\mathbf{k a}}=\sum_{\mu=1}^{d_{n_{0}}} \boldsymbol{\eta}_{\mu}^{n_{0}} \varphi_{\mathbf{k a}}^{\mu n_{0}}$,

as it transforms exactly the same way.

Proof of statement (I)

Let us first focus on a single IR $n_{0}$ with the associated interaction $(g>0)$

$H_{\text {int }}=-g \sum_{\mu, \boldsymbol{k}, \boldsymbol{k}^{\prime}}\left[\psi_{\boldsymbol{k}}^{\dagger} x_{\boldsymbol{k} \mu}^{n_{0}}\left(\psi_{-\boldsymbol{k}}^{\dagger}\right)^{T}\right]\left[\psi_{-\boldsymbol{k}^{\prime}}^{T}\left(X_{\boldsymbol{k}^{\prime} \mu}^{n_{0}}\right)^{\dagger} \psi_{\boldsymbol{k}^{\prime}}\right]$

in the Cooper channel. As we discuss below, making the energetic assumption (i) allows using the weak-pairing description (7). In this limit, we write down the GL expansion to all orders in the order parameter $\tilde{\Delta}_{\boldsymbol{k} a}$ formally expressed in terms of Fermi surface averages $\left\langle\left|\tilde{\Delta}_{\mathbf{k a}}\right|^{2 /}\right\rangle_{a}$. As discussed in more detail in the Supplementary Information S2, resummation up to infinite order shows that $F_{\geq 4}\left[\eta_{\mu}^{n}\right] \geq 0$ and, hence, the superconducting transition must be second order on the mean-field level as long as the normal phase is time-reversal symmetric. This justifies focusing on the first few orders of the GL expansion to deduce constraints on possible pairing states. To fourth order, it holds $F_{\geq 4}=I\left|\boldsymbol{\eta}^{n_{0}}\right|^{4} \beta\left(z^{n_{0}}\right)+\ldots$, where $\boldsymbol{z}^{n_{0}}:=\boldsymbol{\eta}^{n_{0}} /\left|\boldsymbol{\eta}^{n_{0}}\right|, \quad l>0$ is a (temperature-dependent) prefactor, and

$$
\beta\left(\mathbf{z}^{n_{0}}\right)=\sum_{a}\left\langle\left|\sum_{\mu=1}^{d_{n_{0}}} z_{\mu}^{n_{0}} \varphi_{\mathbf{k} a}^{\mu n_{0}}\right|^{4}\right\rangle_{a}
$$

has been introduced. Let us assume that the minimum occurs at $\boldsymbol{z}^{n_{0}} \in \mathbb{R}^{d_{n_{0}}}$ and define $\boldsymbol{z}_{\xi}^{n_{0}}:=\left.\boldsymbol{z}^{n_{0}}\right|_{\boldsymbol{z}_{\mu_{0}}^{n_{0}} \rightarrow z_{\mu_{0}}^{n_{0}} e^{i \xi}}$ for some $\mu_{0}$ with $\boldsymbol{z}_{\mu_{0}}^{n_{0}} \neq 0$. It follows from Eq. (10) that

$\beta\left(\mathbf{z}_{\xi}^{n_{0}}\right) \sim \beta\left(\mathbf{z}^{n_{0}}\right)-C\left(\mathbf{z}^{n_{0}}\right) \xi^{2}$

as $\xi \rightarrow 0$. For $n_{0}$ being a real and multidimensional IR, symmetry properties can be used to show that $C\left(z^{n_{0}}\right)>0$ (see "Methods" section) except for $\boldsymbol{z}^{n_{0}}=\boldsymbol{e}_{\mu_{0}}$, with $\boldsymbol{e}_{\mu}$ denoting the unit vector along the $\mu$ direction, where $C=0$ following from gauge invariance. In the latter case, one has to take instead $\boldsymbol{z}_{\xi}^{n_{0}} \propto$ $\left(\boldsymbol{e}_{\mu_{0}}+i \xi \boldsymbol{e}_{\mu_{1}}\right)$ with $\mu_{1} \neq \mu_{0}$ again yielding $\beta\left(\boldsymbol{z}_{\xi}^{n_{0}}\right)<\beta\left(\boldsymbol{z}^{n_{0}}\right)$ for small, but finite $\xi$. This means that introducing relative complexity between the components lowers the free energy and, hence, the order parameter must break TRS. This means that the two pairing states $e_{(1,0)}$ and $e_{(1,1)}$ in Table 1 are allowed by symmetry but suppressed energetically in the weak-pairing limit. The analogous discussion for complex IRs, which are best thought of as real reducible representations of dimension $2 d_{n_{0}}$, can be found in the Supplementary Information S3. It yields that the superconducting state automatically breaks TRS when $n_{0}$ is complex (also for $\left.d_{n_{0}}=1\right)$. These observations show that having only singly degenerate bands allows for much stronger statements as compared to centrosymmetric systems (see, e.g., the discussion of triplet states in ref. 47).

Two remarks are in order. First, we emphasize that, although the main focus of this paper is on 2D systems, this result also holds for the $3 \mathrm{D}$ case. Second, statement (I) shows that, in the case of singly degenerate Fermi surfaces, the representation of the order parameter being complex or multidimensional is not only a necessary but also a sufficient condition for TRS breaking. Consequently, symmetry-breaking fields (see, e.g., ref. 48) are particularly efficient tools for probing TRS properties in the case of noncentrosymmetric systems.

Symmetry classification of 2D systems

Shifting $\boldsymbol{k} \rightarrow-\boldsymbol{k}$ in the weak-pairing limit and using the behavior of the phase factors in Eq. (6) under this shift, we obtain the important property

$\tilde{\Delta}_{\mathbf{k a}}= \pm \tilde{\Delta}_{-\mathbf{k a}} \quad$ if $\quad \Theta^{2}=\mp 1$.

Naturally, the upper sign is most relevant for fermionic pairing, yet we include the more general behavior for two reasons: Firstly, it illustrates the importance of normal state TRS for the fact that the gap function $\tilde{\Delta}_{\mathbf{k} a}$ has a well-defined parity. Secondly, there are situations ${ }^{49}$ where fermionic TRS is broken, however, the effective low-energy theory of the system has an emergent TRS that satisfies $\Theta^{2}=1$.

Let us first focus on the upper signs in Eq. (12). Suppose that the point group contains a twofold rotation $C_{2}^{\perp}$ with $R_{v}=-1$, i.e., perpendicular to the plane of the system. This is only allowed in even space dimensions, since $\operatorname{det} R_{v}=1$, which is why we will be focusing on 2D systems in the following. As dictated by Eq. (12), $\tilde{\Delta}_{\mathbf{k a}}$ has to be an even function of $\boldsymbol{k}$ and, hence, no solutions with finite gap can occur that are odd under this rotation, which has recently been very clearly explained in ref. 17 .

Proof of statements (II) and (III)

Before discussing below under which conditions we can use the weak-pairing limit (7) to describe superconductivity, let first proceed by proving statement (II) and (III) still assuming that (7) applies. 
To approach (II), we start by considering again the point group $C_{4 v}$ as an example. The order parameter cannot transform under the 2D IR $E$ that is required for TRS-breaking since $E$ is odd under $C_{2}^{\perp}$. Thus, we can exclude a finite order parameter that transforms as $k_{x}+i k_{y}$ or any other superpositions of $k_{x}$ and $k_{y}$ for that matter. In the case of 2D systems, the matrix elements of the pairing field on a non-degenerate Fermi surface are too restrictive to allow for any of these pairing states.

It is straightforward to generalize this analysis to all possible point groups of noncentrosymmetric 2D electron systems: For analogous reasons to $C_{4 v}$, TRS-breaking superconductivity is not possible for the interface point group $C_{4}$. The same holds for the isomorphic groups $D_{4}, D_{2 d}$ and $S_{4}$ that describe possible symmetries of $2 \mathrm{D}$ electronic sheets. For all other symmetry groups without any rotation symmetry (such as for $C_{1}$, i.e. in the absence of any symmetries) or containing only a twofold rotation normal to the plane, all IRs are real and one-dimensional such that TRSbreaking superconductivity is forbidden as well. For the remaining possible noncentrosymmetric point groups of 2D electron systems, all of which contain a threefold rotation, one cannot exclude TRS-breaking superconductivity without further assumptions. This proves statement (II) and shows that it results from the observation that a crystalline point group has a multidimensional (or complex) irreducible representation which is not forced to be odd under $C_{2}^{\perp}$ if and only if it contains a threefold rotation symmetry.

Finally, the general proof of statement (III) requires investigating all double group representations of crystalline point groups (see Supplementary Information S4). For simplicity, we only note that statement (III) is most easily seen to be true in the special case of just a single relevant orbital and when orbital mixing due to spin-orbit coupling can be neglected: In this case, the triplet vector $\boldsymbol{d}$ transforms as a vector under rotation forcing its component along the axis of $C_{2}^{\perp}$ to vanish in any pairing state that is even under $C_{2}^{\perp}$.

\section{Spinless fermions}

Similar reasoning can be applied to the case of spinless fermions, i.e., for the lower signs in Eq. (12). For instance, one can show that, as opposed to (II), the spin-0 TRS must be necessarily broken at the superconducting transition if the point group contains a proper or improper fourfold rotation symmetry. Note that this statement also holds for centrosymmetric point groups as long as the weakpairing description is valid.

\section{Applicability of selection rules}

Let us next discuss under which general conditions the weakpairing description and, hence, the selection rules can be applied. To this end, we derive a general inequality for the zerotemperature condensation energy of a (translation-invariant) superconducting phase with vanishing intra-Fermi-surface matrix elements $\left(D_{\mathbf{k a a}}=0\right)$. As described in more detail in the "Methods" section, we can show that such a state is always energetically disfavored unless the order parameter is larger than (half of) the energetic splitting $E_{\mathrm{so}}$ of the singly degenerate Fermi surfaces. This shows the relation between the validity of the weak-pairing description and assumption (i).

The physical reason is that the superconducting order parameter only couples states at energies differing by $E_{\text {so }}$ as illustrated in Fig. 4 which "cuts off" the conventional Cooper logarithm and, hence, superconductivity can only occur above a threshold coupling strength.

We note that this general result is consistent with the analysis of ref. 45, where a specific, spin-independent electron-electron interaction was considered.

\section{DISCUSSION}

The applications of the symergetic selection rules (I), (II), and (III) to various materials discussed above show that these can both be used to pinpoint the order parameter of $2 \mathrm{D}$ superconductors as well as serve as design principles in the search for superconducting phases with exotic properties such as broken TRS or nontrivial topologies. In this context, it is particularly important that our results are only based on the general assumptions (i) and (ii) and, hence, go beyond specific model studies in noncentrosymmetric systems. ${ }^{24,25,45}$ This has shown which aspects of refs 24 , 25 and 45 are general, i.e., do not depend on microscopic details such as number and character of relevant orbitals or the form of the interaction driving the superconducting instability.

Since inversion symmetry is locally broken at the surface of a material, one might wonder whether the symergetic selection rules are also relevant for the behavior of the superconducting phase at the boundary of the system. In the case of a material such as $\mathrm{Sr}_{2} \mathrm{RuO}_{4}$ which consists of weakly coupled layers as illustrated in Fig. 1C, the condensate near a surface perpendicular to the $z$ axis can be thought of as a set quasi-2D systems with $E_{\mathrm{so}}$ increasing as the distance to the surface is reduced and, hence, bears strong similarities to the superconductor in our gedankenexperiment. In combination with the near degeneracy ${ }^{22}$ with the triplet states transforming under the 1D IRs, we expect the triplet vector to rotate to be parallel to the surface and TRS to be restored locally. While this is the predicted generic behavior of a system with strong spin-orbit coupling and no threefold rotation symmetry, further details of the texture near a surface depend on the microscopic model considered. ${ }^{25}$ It is an interesting open question whether this could account for the absence of magnetic signals in $\mathrm{Sr}_{2} \mathrm{RuO}_{4}{ }^{49}$ that are expected from the chiral $p$-wave nature of the bulk order parameter.

\section{METHODS}

Fourth order of the GL expansion

Let us provide more details on the proof by contradiction based on the fourth order GL expansion first focusing on real IRs. Expanding Eq. (10) with

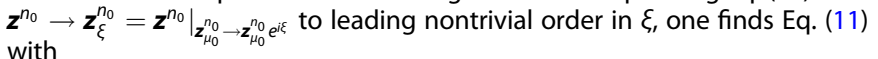

$$
C\left(\mathbf{z}^{n_{0}}\right)=4 \sum_{\mu \neq \mu_{0}}\left(z_{\mu_{0}}^{n_{0}} z_{\mu}^{n_{0}}\right)^{2} \sum_{a}\left\langle\left(\varphi_{\mathbf{k} \mathbf{a}}^{\mu_{0} n_{0}} \varphi_{\mathbf{k} \mathbf{a}}^{\mu n_{0}}\right)^{2}\right\rangle_{a}
$$

To derive Eq. (13), it has been taken into account that $\varphi^{\mu n_{0}} \in \mathbb{R}$ and that the symmetries require the free energy to be invariant under

$\eta_{\mu}^{n_{0}} \rightarrow\left\{\begin{array}{cc}-\eta_{\mu}^{n_{0}}, & \mu=\mu_{0} \\ \eta_{\mu}^{n_{0}}, & \mu \neq \mu_{0}\end{array}, \quad \forall \mu_{0} \in\left\{1,2, \ldots, d_{n_{0}}\right\}\right.$,

for any real IR $n_{0}$ of $2 D$ and $3 D$ point groups. From Eq. (13), we directly see that $C\left(z^{n_{0}}\right)>0$ for all $\boldsymbol{z}^{n_{0}} \neq \boldsymbol{e}_{\mu_{0}}$ unless $\varphi^{\mu n_{0}}=0$. In the latter case, however, the superconducting state is fully ungapped in the weak-pairing limit and, hence, disfavored energetically as discussed in the main text.

If $\boldsymbol{z}^{n_{0}}=\boldsymbol{e}_{\mu_{0}}$, we will use $\boldsymbol{z}_{\xi}=\left(\boldsymbol{e}_{\mu_{0}}+i \xi \boldsymbol{e}_{\mu_{1}}\right) / \sqrt{1+\xi^{2}}$ again yielding Eq. (11), but with modified

$C\left(\mathbf{z}^{n_{0}}\right)=2 \sum_{a}\left[\left\langle\left(\varphi_{\mathbf{k a}}^{\mu_{0} n_{0}}\right)^{4}\right\rangle_{a}-\left\langle\left(\varphi_{\mathbf{k a}}^{\mu_{0} n_{0}} \varphi_{\mathbf{k} \mathbf{a}}^{\mu_{1} n_{0}}\right)^{2}\right\rangle_{a}\right]$,

which is readily shown to be positive (as long as $\varphi^{\mu n_{0}}$ are not identically zero). This completes the proof for the case of real IRs of point groups.

Due to TRS, complex IRs are always degenerate with their conjugate IR and, hence, can be seen as reducible representations of doubled dimension. Being reducible, symmetries are less restrictive in this case and, in particular, Eq. (14) is not guaranteed any more which necessitates a generalized form of the proof presented above. The latter can be found in the Supplementary Information S3.

\section{Inequality for the condensation energy}

To derive a necessary condition for the emergence of a superconducting state with $D_{\text {kaa }}=0$, we consider the most favorable scenario for such a 
pairing state where, at low energies, the effective electron-electron interaction is dominated by the Cooper channel in Eq. (9) with $n_{0}$ being odd under the twofold rotation $C_{2}^{\perp} \in \mathcal{G}$. All other competing channels are assumed to be negligible. From the discussion in the main text, we know that the associated matrix elements $D_{\text {kab }}$ vanish for $a=b$. We analyze whether its zero-temperature condensation energy

$\Delta E\left(\Delta_{0}\right)=\frac{1}{2} \sum_{a=1}^{N} \sum_{\boldsymbol{k}}\left(\left|E_{\mathbf{k a}}\left(\Delta_{0}\right)\right|-\left|\epsilon_{\mathbf{k a}}\right|\right)-\frac{\Delta_{0}^{2}}{2 g}$

is positive for some finite $\Delta_{0}=g\left|\boldsymbol{\eta}^{n_{0}}\right|$. Here $\epsilon_{\mathbf{k a}}$ and $E_{\mathbf{k a}}$ denote the different bands of the normal state and of the superconducting mean-field Hamiltonian, respectively.

To focus on the essential part of the physics, let us consider only $N=2$ singly degenerate bands. Replacing $\left|D_{\boldsymbol{k} 12}\right|$ by its maximum value $\Delta_{0} m$ yields an upper bound $\Delta E^{\max }\left(\Delta_{0}\right)$ on the condensation energy. Physically, it corresponds to the situation of "optimal basis functions" with $\left|D_{\mathbf{k} 12}\right|$ being constant except for negligibly small regions where it has to vanish as dictated by symmetry. Evaluating the sum in Eq. (16) as an integral (cut off energetically at $\Lambda$, constant density of states $\rho_{F}$ ) shows that the condensation energy can only be positive when the spin-orbit splitting $E_{\text {so }}$ on the Fermi surface satisfies

$$
E_{\mathrm{so}}<\frac{2 \Lambda}{\sinh (1 / \lambda)}
$$

where $\lambda=2 \rho_{F} m^{2} g$ denotes the associated dimensionless coupling constant. This means that, in the weak-coupling limit, $\lambda \ll 1$, superconductivity can only emerge when the spin-orbit coupling is exponentially small. We have furthermore found that the value of the order parameter maximizing the energetic gain $\Delta E$ is larger than $E_{\mathrm{so}} / 2$ as stated in the main text.

Model for $\mathrm{Sr}_{2} \mathrm{RuO}_{4}$

To be self contained, we define the model used in the main text to calculate the spectrum of $\mathrm{Sr}_{2} \mathrm{RuO}_{4}$. The centrosymmetric part

$$
\begin{aligned}
h_{\boldsymbol{k}}^{S}= & \left(\begin{array}{ccc}
\epsilon_{x y}(\boldsymbol{k})-\mu-\delta \epsilon_{x y} & 0 & 0 \\
0 & \epsilon_{x z}(\boldsymbol{k})-\mu & t_{\eta} \sin \left(k_{x}\right) \sin \left(k_{y}\right) \\
0 & t_{\eta} \sin \left(k_{x}\right) \sin \left(k_{y}\right) & \epsilon_{y z}(\boldsymbol{k})-\mu
\end{array}\right) \\
& +\frac{\lambda}{2} \sum_{j=x, y, z} L_{j} \cdot \sigma_{j}
\end{aligned}
$$

is taken to be of the form usually applied (see, e.g., ref. 27) to describe the bulk of the material. In Eq. (18), the orbital basis $\left\{4 d_{x y}, 4 d_{x z}, 4 d_{y z}\right\}$ of $\mathrm{Ru}$ orbitals is used and $\sigma_{j}$ are Pauli matrices representing spin. Furthermore, $\quad \epsilon_{x y}(\boldsymbol{k})=-2 t_{3}\left(\cos \left(k_{x}\right)+\cos \left(k_{y}\right)\right)-4 t_{4} \cos \left(k_{x}\right) \cos \left(k_{y}\right)$, $\epsilon_{x z}(\boldsymbol{k})=-2 t_{1} \cos \left(k_{x}\right)-2 t_{2} \cos \left(k_{y}\right)$, and $\epsilon_{y z}(\boldsymbol{k})=-2 t_{2} \cos \left(k_{x}\right)-2 t_{1} \cos \left(k_{y}\right)$. Adding the inversion antisymmetric hopping term $h_{k}^{A}=\delta\left(L_{x} \sin \left(k_{y}\right)-\right.$ $L_{y} \sin \left(k_{x}\right)$ ) already introduced in the main text defines the normal state Hamiltonian $h_{k}=h_{k}^{S}+h_{k}^{A}$ in Eq. (2). To obtain Fig. 2b-d, we have taken $t_{2}=0.1 t_{1}, t_{3}=0.8 t_{1}, t_{4}=0.3 t_{1}, t_{\eta}=-0.04 t_{1}, \lambda=0.2 t_{1}, \mu=t_{1}$ and $\delta \epsilon_{x y}=0.1 t_{1}$ as deduced in ref. 27

\section{ACKNOWLEDGEMENTS}

We thank P. M. R. Brydon and E. J. König for discussions. D.A. is supported by NSF visa grant DMREF 1335215.

\section{AUTHOR CONTRIBUTIONS}

M.S., D.A., and J.S. performed the research and wrote the paper.

\section{COMPETING INTERESTS}

The authors declare that they have no competing interests.

\section{REFERENCES}

1. Reyren, N. et al. Superconducting interfaces between insulating oxides. Science 317, 1196-1199 (2007).

2. Ueno, K. et al. Discovery of superconductivity in $\mathrm{KtaO}_{3}$ by electrostatic carrier doping. Nat. Nanotechnol. 6, 408-412 (2011).

3. Qing-Yan, W. et al. Interface-induced high-temperature superconductivity in single unit-cell FeSe films on $\mathrm{SrTiO}_{3}$. Chin. Phys. Lett. 29, 037402 (2012).
4. Ye, J. T. et al. Superconducting dome in a gate-tuned band insulator. Science $\mathbf{3 3 8}$, 1193-1196 (2012).

5. Han, Y.-L. et al. Two-dimensional superconductivity at (110) $\mathrm{LaAlO}_{3} / \mathrm{SrTiO}_{3}$ interfaces. Appl. Phys. Lett. 105, 192603 (2014).

6. Bernevig, B. A. Topological Insulators and Topological Superconductors (Princeton University Press, 2013)

7. Caviglia, A. D. et al. Electric field control of the $\mathrm{LaAlO}_{3} / \mathrm{SrTiO}_{3}$ interface ground state. Nature 456, 624-627 (2008).

8. Saito, Y. et al. Superconductivity protected by spin-valley locking in ion-gated $\mathrm{MoS}_{2}$. Nat. Phys. 12, 144-149 (2016).

9. Horsdal, M., Khaliullin, G., Hyart, T. \& Rosenow, B. Enhancing triplet superconductivity by the proximity to a singlet superconductor in oxide heterostructures. Phys. Rev. B 93, 220502 (2016).

10. Mannhart, J. \& Schlom, D. G. Oxide interfaces-an opportunity for electronics Science 327, 1607-1611 (2010).

11. Krockenberger, Y. et al. Growth of superconducting $\mathrm{Sr}_{2} \mathrm{RuO}_{4}$ thin films. Appl. Phys. Lett. 97, 082502 (2010).

12. Huth, M. et al. Growth characteristics of sputter-deposited Upt ${ }_{3}$ thin films. J. Phys. 8, 8777-8786 (1996).

13. Altland, A. \& Zirnbauer, M. R. Nonstandard symmetry classes in mesoscopic normal-superconducting hybrid structures. Phys. Rev. B 55, 1142-1161 (1997).

14. Ryu, S., Moore, J. E. \& Ludwig, A. W. W. Electromagnetic and gravitational responses and anomalies in topological insulators and superconductors. Phys. Rev. B 85, 045104 (2012).

15. $\mathrm{Fu}, \mathrm{L}$. Odd-parity topological superconductor with nematic order: application to $\mathrm{Cu}_{x} \mathrm{Bi}_{2} \mathrm{Se}_{3}$. Phys. Rev. B 90, 100509 (2014).

16. Agterberg, D., Brydon, P. \& Timm, C. Inflated nodes in superconductors with broken time-reversal symmetry. arXiv 1608, 06461 (2016).

17. Samokhin, K. V. Symmetry and topology of two-dimensional noncentrosymmetric superconductors. Phys. Rev. B 92, 174517 (2015).

18. Maeno, Y. et al. Superconductivity in a layered perovskite without copper. Nature 372, 532-534 (1994).

19. Ishida, K. et al. Spin-triplet superconductivity in $\mathrm{Sr}_{2} \mathrm{RuO}_{4}$ identified by ${ }^{17} \mathrm{O}$ Knight shift. Nature 396, 658-660 (1998).

20. Kidwingira, F., Strand, J. D., Van Harlingen, D. J. \& Maeno, Y. Dynamical superconducting order parameter domains in $\mathrm{Sr}_{2} \mathrm{RuO}_{4}$. Science 314, 1267-1271 (2006).

21. Xia, J., Maeno, Y., Beyersdorf, P. T., Fejer, M. M. \& Kapitulnik, A. High resolution polar Kerr effect measurements of $\mathrm{Sr}_{2} \mathrm{RuO}_{4}$ : evidence for broken time-reversal symmetry in the superconducting state. Phys. Rev. Lett. 97, 167002 (2006).

22. Rice, T. M. \& Sigrist, M. $\mathrm{Sr}_{2} \mathrm{RuO}_{4}$ : an electronic analogue of ${ }^{3} \mathrm{He}$ ?. J. Phys. 7, L643-L648 (1995).

23. Lax, M. Symmetry Principles in Solid State and Molecular Physics (Wiley, 1974).

24. Tada, Y., Kawakami, N. \& Fujimoto, S. Pairing state at an interface of $\mathrm{Sr}_{2} \mathrm{RuO}_{4}$ : parity-mixing, restored time-reversal symmetry and topological superconductivity. New J. Phys. 11, 055070 (2009).

25. Yanase, Y. Electronic structure and noncentrosymmetric superconductivitity in three-orbital $t_{2 g}$ model with spin-orbit coupling: $\mathrm{Sr}_{2} \mathrm{RuO}_{4}$ near [001] surface/ interface. J. Phys. Soc. Jpn 82, 044711 (2013).

26. Qi, X.-L., Hughes, T. L. \& Zhang, S.-C. Topological invariants for the Fermi surface of a time-reversal-invariant superconductor. Phys. Rev. B 81, 134508 (2010).

27. Scaffidi, T., Romers, J. C. \& Simon, S. H. Pairing symmetry and dominant band in $\mathrm{Sr}_{2} \mathrm{RuO}_{4}$. Phys. Rev. B 89, 220510 (2014).

28. Teo, J. C. Y. \& Kane, C. L. Topological defects and gapless modes in insulators and superconductors. Phys. Rev. B 82, 115120 (2010).

29. Ran, Y., Zhang, Y. \& Vishwanath, A. One-dimensional topologically protected modes in topological insulators with lattice dislocations. Nat. Phys. 5, 298-303 (2009).

30. Schemm, E. R. et al. Evidence for broken time-reversal symmetry in the superconducting phase of $\mathrm{URu}_{2} \mathrm{Si}_{2}$. Phys. Rev. B. 91, 140506 (2015).

31. Schemm, E. R., Gannon, W. J., Wishne, C. M., Halperin, W. P. \& Kapitulnik, A. Observation of broken time-reversal symmetry in the heavy-fermion superconductor Upt $_{3}$. Science 345, 190-193 (2014).

32. Mydosh, J. A. \& Oppeneer, P. M. Colloquium: hidden order, superconductivity, and magnetism: the unsolved case of $\mathrm{URu}_{2} \mathrm{Si}_{2}$. Rev. Mod. Phys. 83, 1301-1322 (2011).

33. Schmalian, J. \& Hübner, W. Nonlinear magneto-optical response of $s$ - and $d$-wave superconductors. Phys. Rev. B 53, 11860-11867 (1996).

34. Kasahara, Y. et al. Superconducting gap structure of heavy-fermion compound $\mathrm{URu}_{2} \mathrm{Si}_{2}$ determined by angle-resolved thermal conductivity. New J. Phys. 11, 055061 (2009).

35. Choi, C. H. \& Sauls, J. A. Identification of odd-parity superconductivity in UPT from paramagnetic effects on the upper critical field. Phys. Rev. Lett. 66, 484-487 (1991).

36. Ohtomo, A. \& Hwang, H. Y. A high-mobility electron gas at the $\mathrm{LaAlO}_{3} / \mathrm{SrTiO}_{3}$ heterointerface. Nature 427, 423-426 (2004). 
37. Herranz, G., Sánchez, F., Dix, N., Scigaj, M. \& Fontcuberta, J. High mobility conduction at (110) and (111) $\mathrm{LaAlO}_{3} / \mathrm{SrTiO}_{3}$ interfaces. Sci. Rep 2, 758 (2012).

38. Annadi, A. et al. Anisotropic two-dimensional electron gas at the $\mathrm{LaAlO}_{3} / \mathrm{SrTiO}_{3}$ (110) interface. Nat. Commun. 4, 1838 (2013).

39. Caviglia, A. D. et al. Tunable Rashba spin-orbit interaction at oxide interfaces. Phys. Rev. Lett. 104, 126803 (2010).

40. Ben Shalom, M., Sachs, M., Rakhmilevitch, D., Palevski, A. \& Dagan, Y. Tuning spin-orbit coupling and superconductivity at the $\mathrm{LaAlO}_{3} / \mathrm{SrTiO}_{3}$ interface: a magnetotransport study. Phys. Rev. Lett. 104, 126802 (2010).

41. Scheurer, M. S. \& Schmalian, J. Topological superconductivity and unconventional pairing in oxide interfaces. Nat. Commun. 6, 6005 (2015).

42. Scheurer, M. S. Mechanism, time-reversal symmetry, and topology of superconductivity in noncentrosymmetric systems. Phys. Rev. B 93, 174509 (2016).

43. Liu, D. et al. Electronic origin of high-temperature superconductivity in singlelayer FeSe superconductor. Nat. Commun. 3, 931 (2012)

44. Sigrist, M. \& Ueda, K. Phenomenological theory of unconventional superconductivity. Rev. Mod. Phys. 63, 239-311 (1991).

45. Frigeri, P. A., Agterberg, D. F., Koga, A. \& Sigrist, M. Superconductivity without inversion symmetry: MnSi versus $\mathrm{CePt}_{3}$ Si. Phys. Rev. Lett. 92, 097001 (2004).
46. Sergienko, I. A. \& Curnoe, S. H. Order parameter in superconductors with nondegenerate bands. Phys. Rev. B 70, 214510 (2004).

47. Blount, E. Symmetry properties of triplet superconductors. Phys. Rev. B 32, 2935-2944 (1985).

48. Hicks, C. W. et al. Strong increase of $T_{\mathrm{c}}$ of $\mathrm{Sr}_{2} \mathrm{RuO}_{4}$ under both tensile and compressive strain. Science 344, 283-285 (2014).

49. Kirtley, J. R. et al. Upper limit on spontaneous supercurrents in $\mathrm{Sr}_{2} \mathrm{RuO}_{4}$. Phys. Rev. B 76, 014526 (2007)

(i) This work is licensed under a Creative Commons Attribution 4.0 article are included in the article's Creative Commons license, unless indicated otherwise in the credit line; if the material is not included under the Creative Commons license, users will need to obtain permission from the license holder to reproduce the material. To view a copy of this license, visit http://creativecommons.org/licenses/by/4.0/

(c) The Author(s) 2017

Supplementary Information accompanies the paper on the npj Quantum Materials website (doi:10.1038/s41535-016-0008-1). 